[Vicino Oriente XIX (2015), pp. 51-64]

\title{
UNA MATRICE PER TERRECOTTE CON SILENO DALL'AREA SACRA DEL KOTHON A MOZIA
}

\author{
Nicola Chiarenza - Sapienza Università di Roma
}

During the XXV season of excavations carried out by Rome «La Sapienza» University Expedition to Motya, a terracotta mould was found in the Sacred Area of the Kothon. The mould shows a quarter of a silenus bringing the handle of a vase. The iconographic analysis and the stylistic parallels allow to date the mould and to set it in the cultural context of Motya. The iconological analysis suggests the possible relations between the subject of the mould and the deities worshipped in the Sacred Area of the Kothon.

Keywords: terracotta mould; silenus; wine; Sacred Area of the Kothon; Motya

\section{PREMESSA}

In occasione della XXV (2005) campagna di scavi condotta a Mozia dalla Missione Archeologica dell'Università di Roma «La Sapienza» ${ }^{1}$, nel settore C Nord dell’Area sacra del Kothon ${ }^{2}$ è stata rinvenuta una matrice per terrecotte frammentaria. Il reperto si trovava all'esterno del Tempio di Astarte $^{3}$ (fig. 1), in un contesto successivo alla frequentazione cultuale dell'area sacra ${ }^{4}$.

In questa sede, dopo la descrizione della matrice, l'individuazione di confronti e l'inquadramento cronologico, verranno proposte alcune considerazioni di ordine iconografico e iconologico.

1 Sono grato al Professor Lorenzo Nigro per avermi dato l’opportunità di studiare e pubblicare il reperto che viene qui presentato. La presente nota trae spunto da uno studio condotto da chi scrive sulla coroplastica votiva rinvenuta nelle campagne di scavo XXV-XXVIII (2005-2008) nell'Area sacra del Kothon, di prossima pubblicazione all'interno del volume Mozia XIV.

2 Le ricerche nel settore C Nord sono state coordinate sul campo dalla dott.ssa Daria Montanari. I resoconti degli scavi condotti nella Zona C da parte della Missione archeologica dell’Università di Roma "La Sapienza" a partire dal 2002 sono stati pubblicati, oltre che in numerose riviste scientifiche, nei rapporti preliminari della serie Quaderni di Archeologia Fenicio Punica (Nigro ed. 2004, 33-138; ed. 2005). Sull’Area sacra del Kothon si vedano da ultimo: Nigro 2014a; 2014b; 2015, 83-91, 93-95 (con bibliografia).

3 Dell'edificio sacro, posto circa $20 \mathrm{~m}$ a nord del Tempio del Kothon, sono state individuate due ricostruzioni successive (C6 e C4). Sulla base dell'orientamento della facciata e della nicchia all'interno della cella e di un ostrakon con l'epiteto AGLAIA, l'edificio è stato attribuito ad Astarte (Nigro 2015a, 93-95, figg. 14-15). Per le testimonianze dei culti tributati a Mozia a questa divinità e per gli edifici ad essa dedicati si vedano: Amadasi Guzzo 1981; Nigro 2010b; 2015b; Spagnoli 2013.

4 La matrice, attribuita all’US.1724, si trovava tra il muro del Temenos Circolare e il lato orientale del sacello, ai margini della fossa F.1727, praticata sul rifacimento più recente del muro di temenos. Potrebbe trattarsi pertanto di un reperto di risulta da strati più antichi intaccati dalla fossa. 


\section{LA MATRICE MC.05.396: ANALISI ICONOGRAFICA}

Il reperto raffigura in negativo la metà superiore sinistra di un sileno (fig. 2) ${ }^{5}$. Lo stato non usurato della matrice, l'accurata caratterizzazione del viso e la realizzazione di un positivo in gesso ${ }^{6}$ consentono di distinguere l'arcata sopracciliare prominente, la palpebra superiore appena rilevata rispetto al maggiore spessore di quella inferiore, il naso dai tratti morbidi sopra i folti baffi; la lunga barba, dal profilo appuntito, resa con sottili linee ondulate è separata, mediante una linea netta e profonda, dallo zigomo prominente; dell'orecchio, si riconoscono il profilo esterno arrotondato e la conca al centro del padiglione. La parte conservata del busto è nuda. Il braccio sinistro è aderente al fianco, mentre la mano chiusa ${ }^{7}$ trattiene l'ansa di un vaso potorio o di un cratere.

\section{CONFRONTI E INQUADRAMENTO CRONOLOGICO}

Tale iconografia ${ }^{8}$ costituisce una delle varianti di terrecotte raffiguranti sileni accovacciati, sovente itifallici, prodotte a partire dal VI secolo a.C. ${ }^{9}$ In particolare, fra i sileni che trattengono un vaso, una statuetta conservata a Berlino (fig. 3), sul cui contesto di rinvenimento (verosimilmente in Italia) non si possiedono dati, costituisce il confronto più pertinente per la matrice MC.05.396, mentre un ulteriore parallelo potrebbe essere riconosciuto in una terracotta proveniente da Agrigento ${ }^{10}$.

Una variante dello stesso tema è individuabile in due terrecotte da Roma, datate agli inizi del IV secolo a.C. ${ }^{11}$, le quali si discostano dalla matrice moziese per la diversa caratterizzazione del viso e per la maggiore prossimità del vaso alla bocca (fig. 4).

Il tema perdura, con alcune variazioni, tra il IV e il III secolo a.C. anche in ambito punico, come testimonia una terracotta da Cartagine raffigurante un sileno accovacciato che trattiene per le anse un cratere posto fra le gambe ${ }^{12}$ (fig. 5). I particolari del volto, tuttavia, distinguono la statuetta cartaginese dalla matrice MC.05.396.

5 La matrice ha sezione semicircolare con superficie interna concava. Produzione: non locale. Altezza conservata: 7,4 cm; larghezza conservata: 5,9 cm; spessore: 1,4 cm; peso: 75,2 g. Argilla depurata; colore corpo: 5YR6/8 Reddish Yellow. Della figura si conservano la testa (ad eccezione della sommità) e il busto.

6 Desidero esprimere un ringraziamento sentito al Maestro Stefano Ferrari dell'Istituto Centrale per il Restauro di Roma che ha realizzato il calco.

7 La prominenza semicircolare sotto la mano potrebbe essere attribuita al ginocchio.

8 Il sileno con kantharos è attestato, nel V sec. a.C., anche su vasi metallici di produzione etrusca (Besques 1982, 269, fig. 9).

9 Alcuni esemplari sono in Winter 1903a, 216-218. Per le terrecotte raffiguranti sileni itifallici è stata ipotizzata un'origine in area ionica (Blinkenberg 1931, 561-564, nn. 2319-2329, tavv. 108-109) o più in particolare a Rodi (Higgins 1954, 73-74, nn. 159-165, tav. 31; Moscati 1987a, 12) intorno alla fine del VI secolo a.C. Per quanto riguarda la Sardegna punica due sileni itifallici provengono da Tharros (Higgins 1954, 74, n. 164, tav 31:164; Moscati 1987a, 12-13, tav. I:A1). Una terracotta di sileno accovacciato datata al V secolo a.C., non distante dalla matrice moziese per la composizione della figura, proviene dalla tomba 16 della necropoli occidentale di Himera (Vassallo 2005, 79, fig. 127).

10 Per l'esemplare a Berlino si veda Winter 1903a, 218, n. 2, dove viene anche proposto il confronto con la statuetta da Agrigento. Per quest'ultima, la raffigurazione grafica verosimilmente non realistica (Schiavo 1756, 71-75) induce a proporre con cautela il confronto con la matrice moziese.

11 Winter 1903b, 393, n.4; Besques 1982, 269-270, fig. 10.

12 Cherif 1997, 73-74, n. 231, tav. XXVII. 
La coppa sotto il mento e un volto confrontabile con quello dell'esemplare cartaginese si ritrovano in una statuetta proveniente dall'insediamento punico di Selinunte. Il reperto, databile tra la seconda metà del IV e la prima metà del III secolo a.C., appartiene a quella particolare classe di terrecotte forse utilizzate come thymiateria e note con la definizione di sileno-fiore ${ }^{13}$.

In altre tipologie di reperti fittili possono essere riconosciuti dei confronti pertinenti per il volto del sileno raffigurato nella matrice MC.05.396 e utili al suo inquadramento cronologico. Il primo è individuabile nella stessa isola, in una terracotta di incerta funzione (sostegno?), datata agli inizi del V secolo a.C. L'oggetto ${ }^{14}$, di produzione locale, raffigura la testa di un sileno che per le caratteristiche del volto è accostabile al personaggio della matrice oggetto di studio (fig. 6).

Confronti stilistici stringenti sono inoltre ravvisabili nelle antefisse sileniche di Tipo B (V secolo a.C.) della classificazione realizzata da P. Pelagatti ${ }^{15}$. Fra queste, le antefisse appartenenti alla variante B3 mostrano una caratterizzazione dei tratti somatici (in particolare degli occhi e della barba) assai prossima a quella della terracotta moziese, come dimostra un'antefissa da $\operatorname{Naxos}^{16}$ (fig. 7).

Sulla base dei confronti individuati è pertanto possibile proporre per la matrice MC.05.396 una datazione nel V secolo a.C., presumibilmente nella prima metà, senza dimenticare la prolungata persistenza delle iconografie nella coroplastica ${ }^{17}$.

\section{RECEPIRE E RIPRODURRE: LA MATRICE NEL CONTESTO MOZIESE}

La produzione locale di terrecotte di tradizione ellenica (mediante matrici importate o prodotte sull'isola da originali greci) è un fenomeno diffuso a Mozia ${ }^{18}$, ma sono minoritari i casi in cui disponiamo di tali matrici ${ }^{19}$. La realizzazione in loco, così come l'importazione

13 Cavallari 1884, 323, tav. V:441, 489; Bisi 1968, 44, figg. 6-7; Spatafora - Gandolfo 2014, 53. Sono attestate numerose varianti fra le terrecotte di sileno-fiore; alcuni di queste provengono da Morgantina (Bell 1981, 233, n. 932, tav. 138:932) e Capua (Besques 1982, 268, figg. 6-8. L'editore di questa terracotta la ritiene una comune statuetta; la presenza di un foro circolare sulla sommità della testa, forse destinato ad alloggiare il calice del fiore, potrebbe consentire di attribuire il reperto alla tipologia dei sileni-fiore).

14 La terracotta è stata rinvenuta nella porzione di abitato messa in luce nella cosiddetta Zona $\mathrm{E}$ (Famà - Toti 2000, 456-457, 467, n. 16, tav. XC:1)

15 Pelagatti 1965. Il Tipo B si discosta dal tipo A per la perdita dei tratti ferini del sileno.

16 Epifanio 1990, 104, 254-255, n. 91. Sono state sottolineate analogie formali e stilistiche tra le antefisse sileniche di Naxos e le serie monetali con Dioniso e il sileno, coniate dalla stessa colonia nella prima metà del V secolo a.C. (da ultimo Albertocchi 2012, 157). È interessante notare che sul rovescio dei tetradrammi appartenenti a questa serie è raffigurato un sileno itifallico accosciato che impugna con la mano sinistra un kantharos (Garraffo 1990, 155-156).

17 Riguardo alla pericolosità di datazioni su base esclusivamente stilistica per le terrecotte si leggano i moniti espressi da J. Uhlenbrock in merito alle produzioni coroplastiche in Sicilia tra il V e il IV secolo a.C. (Uhlenbrock 2002, 321-326).

18 Bisi 1990, 26-27; Ciasca 1991b, 19; Mammina - Toti 2011, 33, 35.

19 Alla Collezione Whitaker, esposta nell'omonimo museo dell'isola, appartiene una matrice di protome proveniente dall'area di Porta Nord (ringrazio la dott.ssa M.P. Toti per la notizia). Due frammenti di matrici di produzione moziese, provenienti dalla cosiddetta area industriale nota come Zona K, raffigurano Eracle e l'idra (Falsone 1989, 61, fig. 22) e una figura di tipo Medma seduta su un trono con zampe leonine (Mammina - Toti, 2011, 34, fig. 10). Una matrice raffigurante un sileno con tirso proviene da uno scarico realizzato nel 
di matrici e terrecotte greche, testimonia da un lato l'acquisizione stabile di iconografie elleniche nel repertorio moziese ${ }^{20}$, dall'altro il ruolo svolto dall'isola in parte nella rielaborazione e soprattutto nella diffusione nel restante mondo punico di tali iconografie provenienti sia da ambiente siceliota, che magnogreco ${ }^{21}$. In riferimento a tali dinamiche devono essere valutati, pertanto, il tema e l'iconografia della matrice MC.05.396 che potrebbero essere anche alla base della presenza di analoghe realizzazioni attestate in altre aree puniche, quale quella ibicenca (fig. 8) ${ }^{22}$.

\section{OLTRE L'ICONOGRAFIA: IL SILENO, IL VINO E LE DIVINITÀ DELL'AREA SACRA DEL KOTHON}

Se il rinvenimento a Mozia di una matrice con iconografia ellenica non suscita pertanto meraviglia, più complessa risulta l'individuazione dei motivi che portarono alla scelta, all'interno dell'Area sacra del Kothon, del tema raffigurato nella matrice MC.05.396.

A tale proposito, non si può fare a meno di ricordare che vi sono molti elementi in comune tra la figura del sileno e quella di Bes e che in alcuni casi la sovrapposizione tra le due figure passava attraverso temi e ambientazioni legate al vino ${ }^{23}$, elemento richiamato dalla coppa o dal cratere che il sileno raffigurato nella matrice stringe tra le mani. I due personaggi, inoltre, sono entrambi connessi agli ambiti dell'erotismo e della fecondità tutelati da Astarte, alla quale era consacrato l'omonimo tempio.

L’iconografia del sileno (itifallico?), uno dei partecipanti al tiaso dionisiaco, potrebbe anche essere dovuta alla stretta relazione tra Afrodite ${ }^{24}$ e Dioniso, divinità legata alla fecondità del suolo e degli esseri viventi ${ }^{25}$.

IV secolo a.C. sulla strada messa in luce nel settore di abitato noto come Zona B (Toti 2008, 77-78, fig. 10). Gli scavi ivi condotti nel 1989 e nel 1991 da Maria Luisa Famà (Soprintendenza di Trapani) e Antonia Ciasca (Università di Roma «La Sapienza») sono ancora inediti (un quadro sintetico è offerto in Famà 2002, 28). Le ricerche nella zona B sono riprese nel 2008 da parte della Missione archeologica di Roma «La Sapienza» (Nigro 2012b, 210). Infine una matrice per protomi femminili è stata rinvenuta nel 2013 dagli scavi condotti dalla Missione archeologica di Roma «La Sapienza» presso il Santuario C7, nel settore occidentale del Temenos Circolare (notizie preliminari sul Santuario C7 sono in Nigro 2011). Per il reperto è stato proposto un confronto con una protome da Selinunte appartenente al tipo Wiederkeher Schuler 7E (Spagnoli 2013, 159). A giudizio di chi scrive, tuttavia, la matrice MC.13.100 è attribuibile ai Tipi C o D del Gruppo 10 (530510 a.C. ca.) della tipologia realizzata da E. Wiederkeher Schuler (2004, 79-80, 180-181, tav. 43) per le protomi del Santuario della Malophoros a Selinunte.

20 L'ellenizzazione della cultura moziese, i cui primi segnali sono riconoscibili già nel VI secolo a.C., acquista proporzioni notevoli nel corso del secolo successivo. Oltre che nella produzione coroplastica, il fenomeno è riscontrabile in altri ambiti. Sull'ellenizzazione del repertorio vascolare moziese si vedano: Ciasca 1991a, 183-185; Orsingher 2011, 114, 128-129; Giardino 2013.

21 Moscati 1987b, 154-155; Bisi 1990, 34; Ciasca 1991b, 13; Uberti 1997, 195-196.

22 Si tratta della matrice di testa maschile barbuta riproposta in questa sede e di una placca rettangolare con sileno itifallico (Almagro Gorbea 1980, 274-275, tav. CCV). Sulla coroplastica ibicenca che in alcuni casi recepisce senza modifiche temi e iconografie elleniche e in altri le rielabora con un linguaggio locale si veda Bisi 1988, 345-346.

23 Molteplici sono gli aspetti comuni alle due figure (Jesi 1962, 263-275). In particolare esse condividono la partecipazione ai temi dell'ebbrezza, della gioia e dell'erotismo; sileno, come accompagnatore di Dioniso, Bes come partecipante al corteggio della dea Hathor (Capriotti Vittozzi 2007, 102).

24 Per i rapporti tra la figura di Afrodite e quella di Astarte si veda Bonnet 1996, 19, 147-150. 
Tuttavia, il rinvenimento della matrice al di fuori dell'edificio e in un contesto successivo all'abbandono dell'Area sacra induce a considerare con cautela una relazione diretta tra il tema iconografico della matrice e i culti praticati nel sacello.

Se si vuole analizzare tale tema in relazione non solo al Tempio di Astarte, ma all'intera Area sacra del Kothon, occorre ricordare che Dioniso, quale dio della vegetazione ${ }^{26}$, condivideva il carattere infero e ctonio con Demetra ${ }^{27}$.

Inoltre, l'identificazione tra Dioniso e Osiride ${ }^{28}$ (equivalente egiziano di Orione), menzionata da Erodoto, appare suggestiva alla luce degli elementi presenti nel Tempio del Kothon che potrebbero essere ricondotti ad Orione ${ }^{29}$.

Il vino, infine, sembra aver rivestito un ruolo importante in alcune pratiche rituali presso installazioni e apprestamenti connessi con il mondo infero, sia nel Tempio del Kothon che in altri settori del Temenos Circolare ${ }^{30}$.

La lettura iconologica che è stata suggerita potrebbe trovare conferma in un altro reperto che attesta la presenza del sileno all'interno del Temenos Circolare, in relazione con il vino e con l'ambito ctonio. Si tratta di un sileno raffigurato sul fondo interno di una coppa attica a figure rosse, deposta alla fine del V secolo a.C. nel rituale di obliterazione del Pozzo

25 Tali ragioni sono alla base dell'unione tra il dio e Afrodite o, in altri casi, della concezione di Dioniso come figlio della dea dell'amore (Gasparri - Veneri 1986, 415-416, 418).

26 Uno degli aspetti più importanti del dio, inoltre, è la sua caratterizzazione come divinità dell'elemento umido e della natura rigogliosa (Gasparri - Veneri 1986, 415), elementi che sin dalle prime frequentazioni caratterizzavano l'Area sacra del Kothon, sorta nei pressi di uno stagno alimentato da diverse sorgenti di acqua dolce (Nigro - Spagnoli 2012, 2-11; Nigro 2012a, 301-303; Nigro 2014a, 495; Nigro 2014b, 2-3, 8-10). L'appartenenza del sileno al corteggio di Dioniso non è tuttavia sufficiente a ipotizzare la presenza di culti dionisiaci nell'Area sacra del Kothon. Non poche sono le attestazioni dall'area moziese di personaggi appartenenti al corteggio dionisiaco. Oltre le terrecotte di sileni già citate nelle note 14 e 19 due ulterior esempi di plastica vascolare raffiguranti tali personaggi sono in De Vita 2011, 18, 21, figg. 18-19, 22. A questi si possono aggiungere alcune scene di ambito dionisiaco attestate nella ceramica a figure nere e a figure rosse rinvenuta sull'isola (Nigro ed. 2007, 76, 250, 252, tavv. LXVIII-LXIX; Nigro ed. 2011, 300, MF.05.1278/4, tav. LXXXV). Tali elementi non bastano comunque a provare l'esistenza di culti dionisiaci a Mozia, tema da affrontare con cautela nonostante le ipotesi di P. De Vita (2011) che ha interpretato secondo questa chiave di lettura alcuni reperti moziesi di ambiti e cronologia differenti.

27 In Pindaro inoltre il dio è considerato paredro di Demetra (Gasparri - Veneri 1986, 416). A questa dea, o a divinità femminili dalle caratteristiche simili non appartenenti al pantheon greco, possono essere ricondotti una serie di rinvenimenti dall'Area sacra del Kothon, fra i quali una terracotta frammentaria raffigurante una mano con melagrana (Spagnoli 2013, 159). Di recente è stato proposto di attribuire a Demetra, o ad una interpretatio demetriaca di Astarte, alcuni dei reperti consacrati nei rituali di obliterazione di due pozzi del settore sud-occidentale dell'Area sacra del Kothon (Spagnoli 2013, 158-160).

28 Tale identificazione risalirebbe almeno al V secolo a.C. In seguito, in Diodoro, Dioniso è riconosciuto come figlio di Zeus/Osiride e Io/Iside (Gasparri - Veneri 1986, 417). Per l’identificazione tra Iside e Astarte si veda Bonnet 1996, 19, 63-67.

29 Nigro 2009, 262-265; Nigro 2010a, 21-22.

30 Diversi apprestamenti connessi con il sottosuolo caratterizzavano il Tempio del Kothon in tutte le fasi monumentali: il Tempio C5 della fine dell’VIII-VII secolo a.C. (Nigro 2014a, 496, fig. 6) il Tempio C1 della seconda metà del VI secolo a.C. (Nigro 2012a, 298), il Tempio C2 del V secolo a.C. (Nigro 2012a, 299, fig.

11). Vino e altre offerte attribuite a Baal 'Addir venivano consacrate in apprestamenti connessi con il mondo infero anche nel settore sud-occidentale del Temenos Circolare (Nigro - Spagnoli 2012; Nigro 2014a, 493494; Spagnoli 2013, 155-160) 
P.1660, un apprestamento attraverso il quale venivano dedicate offerte ad una coppia divina che potrebbe essere riconosciuta come Baal 'Addir/Astarte (Poseidon/Demetra) ${ }^{31}$.

\section{CONSIDERAZIONI CONCLUSIVE}

La datazione e la lettura iconologica della matrice MC.05.396 proposte in questa sede si inseriscono in un contesto, quello del V secolo a.C., nel quale l'ellenizzazione della cultura moziese non è riconoscibile solo negli aspetti iconografici ma mostra i propri effetti anche in ambito cultuale e verosimilmente rituale.

Il personaggio raffigurato, anche grazie al vaso che recava fra le mani, può essere messo in relazione, più o meno diretta, sia con gli ambiti presieduti dalle divinità femminili attestate nell'Area sacra del Kothon (Astarte e Demetra) sia con la divinità maschile (Baal) e più in generale con il mondo ctonio al quale questo luogo di culto era connesso.

Le future ricerche e l'analisi diacronica di un maggior numero di dati provenienti dall'area sacra consentiranno, forse, di comprendere se il personaggio raffigurato nella matrice MC.05.396 avesse un'identità ben precisa e unanimemente riconosciuta ${ }^{32}$ e di precisare meglio la relazione tra questo tema iconografico e le divinità alle quali era tributato il culto all'interno dell'Area sacra del Kothon. Un luogo nel quale, alla luce delle ricerche recenti, si sovrapposero esigenze comuni alle diverse tradizioni culturali della regione ${ }^{33}$

31 La coppa (MC.11.4512/2) è datata alla seconda metà del V secolo a.C. Particolare importanza acquista la presenza, sul fondo esterno del vaso, di una iscrizione dedicatoria incisa, composta dalle lettere fenicie lamed e alef (Nigro - Spagnoli 2012, 51, 53, 57, fig. 35, tav. V; Spagnoli 2013, 154-158). Degna di nota appare la possibilità che anche in questo contesto, possa essere istituita un'associazione tra la figura del sileno (o Bes?) e la dea Astarte.

32 La questione delle divinità che i frequentatori dei luoghi di culto moziesi riconoscevano nelle terrecotte di iconografia ellenica non è di facile soluzione. Ad una concezione schematica secondo la quale in età arcaica $\mathrm{e}$ classica ciascuna terracotta con iconografia ellenica veniva identificata con una divinità fenicia, mentre nel IV secolo a.C. divinità e ritualità greche si sovrapponevano a quelle fenicie (Bisi 1990, 35), sarebbe da preferire un approccio problematico che tiene conto dei cambiamenti nel tempo ma non esclude la possibilità di fenomeni di sincretismo e interpretatio già in età arcaica o di persistenze ancora nel IV e nel III secolo a.C. Tali problematiche sono state affrontate da chi scrive a margine dello studio già citato di prossima pubblicazione. Questi temi sono molto attuali anche negli studi etruscologici. A proposito del Santuario di Pyrgi si veda ad esempio: Belelli Marchesini- Carlucci - Gentili - Michetti 2012.

33 Nigro - Spagnoli 2012, 32, 50, 57; Spagnoli 2013, 159-160; Nigro 2015, 96-97. 


\section{BIBLIOGRAFIA}

AlBertocChi, M.

2012 La coroplastica siceliota nella prima metà del V sec. a. C.: M. Albertocchi - A. Pautasso (a cura di), Philotechnia. Studi sulla coroplastica della Sicilia greca (Monografie dell'Istituto per i Beni Archeologici e Monumentali 5), Catania 2012, pp. 141-161.

Almagro Gorbea, M.J.

$1980 \quad$ Corpus de las terracottas de Ibiza (Bibliotheca praehistorica Hispana 18), Madrid 1980. AmADASI GuZzo, M.G.

1981 Culti femminili a Mozia: Rivista di Studi Fenici 9 (1981), Supplemento, pp. 7-11.

Belelli Marchesini, B. - Carlucci, C. - Gentili, M. D. - Michetti, L.

2012 Riflessioni sul regime delle offerte nel Santuario di Pyrgi: G.M. DeLla Fina (a cura di), Il Fanum Voltumnae e i santuari comunitari dell'Italia antica. Atti del XIX Convegno Internazionale di Studi sulla Storia e l'Archeologia dell'Etruria (Annali della Fondazione

BELL, M. per il Museo «Claudio Faina», volume XIX), Roma 2012, pp. 227-263.

1981 Morgantina Studies I. The Terracottas, Princeton 1981.

BESQUES, S.

1982 La représentation du Silène au canthare: Revue archéologique 1982, 2, pp. 263-272.

BISI, A.M.

1968 Influenza della coroplastica siceliota sulla produzione punica: Sicilia Archeologica 3 (1968), pp. 41-44.

1988 Le terrecotte figurate: S. Moscati (a cura di), I Fenici, Milano 1988, pp. 328-353.

1990 Le terrecotte figurate fenicie e puniche in Italia (Itinerari V), Roma 1990.

BLINKENBERG, C.

$1931 \quad$ Lindos. Fouilles de l'Acropole, 1902-1914, I. Les petites objets, Berlin 1931.

BONNET, C.

1996 Astarté. Dossier documentaire et perspectives historiques (Contributi alla Storia della Religione Fenicio-Punica II), Roma 1996.

CAPRIOTTI VITTOZZI, G.

2007 Rivisitando la tomba di Petosiri: note su alcuni aspetti iconografici: Vicino Oriente XIII (2007), pp. 101-113.

CAVALLARI, F.S.

1884 Selinunte: Notizie degli Scavi di Antichità 1884, pp. 318-325.

CHERIF, Z.

1997 Terres cuites puniques de Tunisie (Corpus delle antichità fenicie e puniche), Roma 1997.

CIASCA, A.

1991a La ceramica fenicia di Sicilia e i suoi rapporti con le produzioni coeve: I vasi attici ed altre ceramiche coeve in Sicilia. Atti del convegno internazionale, Catania, Camarina, Gela, Vittoria, 28 marzo - 1 aprile 1990, volume secondo (Cronache di Archeologia 30), Catania 1991, pp. 179-186.

1991b Protomi e maschere puniche (Itinerari VII), Roma 1991.

DE VITA, P.

2011 Culti dionisiaci a Mozia: E. ACQUARO - G. SAvio (a cura di), Scavi e ricerche a Mozia II, Lugano 2011, pp. 13-23. 
EPIFANIO, E.

1990 La decorazione fittile architettonica: Lo stile severo in Sicilia. Dall'apogeo della tirannide alla prima democrazia, Museo Archeologico Regionale, Palermo 10 febbraio - 30 settembre 1990, Palermo 1990, pp. 101-105.

FALSONE, G.

1989 Mozia, Zona K. La Quarta campagna di scavo: Sicilia Archeologica 71 (1989), pp. 51-63. FAMÀ, M.L.

2002 L’abitato: M.L. FAMÀ, (a cura di), Mozia. Gli scavi nella “Zona A” dell'abitato (Collana di Archeologia del Centro Internazionale di Studi Fenici, Punici e Romani, Comune di Marsala 1), Bari 2002, pp. 23-34.

FAMÀ, M.L. - TOTI, M.P.

2000 Materiali dalla 'Zona E' dell'abitato di Mozia. Prime considerazioni: Atti delle Terze Giornate Internazionali di Studi sull’Area Elima, Gibellina - Erice - Contessa Entellina, 23-26 ottobre 1997, Pisa - Gibellina 2000, pp. 451-478.

GARRAFFO, S.

1990 Tipi monetali di stile severo: Lo stile severo in Sicilia. Dall'apogeo della tirannide alla prima democrazia, Museo Archeologico Regionale, Palermo 10 febbraio - 30 settembre 1990, Palermo 1990, pp. 151-157.

GASPARRI, C. - VENERI, A.

1986 Dionysos: Lexicon Iconographicum Mytologie Classicae III, Zürich - München 1986, pp. 414-514.

GIARDINO, S.

2013 La ceramica comune tra VI e V secolo a.C. dai recenti scavi a Mozia: dal repertorio originariamente fenicio all'influenza della tradizione greca: L. GIRÓN - M. LAZARICH - M. CONCEIÇÂO (eds.), Actas del I Congreso Internacional sobre Estudios Cerámicos. Homenaje a la dra. Mercedes Vegas, Cádiz 1 al 5 de noviembre de 2010, Cádiz 2013, pp. 835-859.

HIGGINS, R.A.

1954 Catalogue of the Terracottas in the Department of Greek and Roman Antiquites, British Museum. Vol. I, Greek: 730-330 B.C., London 1954.

JESI, F.

1962 Bes e sileno: Aegyptus 42/3-4 (1962), pp. 257-275.

MAMMinA, G. - TоTI, M.P.

2011 Considerazioni sulla coroplastica votiva del tofet di Mozia (Marsala-Italia): Sardinia Corsica et Baleares Antiquae 9 (2011), pp. 31-50.

MOSCATI, S.

1987a Iocalia punica. La collezione del Museo Nazionale G. A. Sanna di Sassari: Atti della Accademia Nazionale dei Lincei. Classe di Scienze morali, storiche e filologiche. Memorie, Serie VIII/29 (1987), pp. 1-149.

1987b L'arte della Sicilia punica, Milano 1987.

NigRO, L.

2009 Il Tempio del Kothon e il ruolo delle aree sacre nello sviluppo urbano di Mozia dall’VIII al IV sec. a.C.: S. HeLAS - D. MARzOLI (Hrsg.), Phönizisches und punisches Städtewesen. Akten der internationalen Tagung in Rom vom 21. bis 23. Februar 2007 (Iberia Archaeologica 13), Mainz am Rhein 2009, pp. 241-270.

2010a L'orientamento astrale del Tempio del Kothon di Mozia: E. ANTONELLo (a cura di), Il cielo e l'uomo: problemi e metodi di astronomia culturale. Atti del VII Convegno Nazionale della Società Italiana di Archeoastronomia, Roma, Museo Nazionale Romano, Terme di Diocleziano, 28-29 settembre 2007, Roma 2010, pp. 15-24. 
2010b Il Sacello di Astarte e i culti femminili a Mozia: G. BARTOLONI - P. MATthiAe - L. NigRo L. Romano (a cura di), Tiro, Cartagine, Lixus: nuove acquisizioni. Atti del Convegno Internazionale in onore di Maria Giulia Amadasi Guzzo, Roma 24-25 novembre 2008 (Quaderni di Vicino Oriente IV), Roma 2010, pp. 163-180.

2011 Mozia 2011. Rapporto preliminare sulla XXI campagna di scavi, prospezioni e restauri dell’Università di Roma "La Sapienza", http://www.lasapienzamozia.it/mozia/risultatidegli- scavi/recenti-scoperte-della-missione/fast-report-2011.

2012a The Temple of the Kothon at Mozia, Sicily: Phoenician Religious Architecture from the Levant to the West: M. Gruber - S. Ahituv - G. Lehmann - Z. TAlshir (eds.), All the Wisdom of the East. Studies in Near Eastern Archaeology and History in Honor of Eliezer D. Oren (Orbis Biblicus et Orientalis 255), Fribourg - Göttingen 2012, pp. 293-331.

2012b Scavi e restauri dell’Università di Roma 'La Sapienza’ a Mozia, 2007-2009: il Tempio del Kothon, il Temenos Circolare, il Sacello di Astarte e il Tofet: C. Ampolo (a cura di), Agora, foro e istituzioni politiche in Sicilia e nel Mediterraneo antico. Atti delle settime giornate internazionali di studi sull'area elima e la Sicilia occidentale nel contesto del Mediterraneo, Erice 12-15 ottobre 2009, Pisa 2012, pp. 207-218.

2014a Il primo stanziamento fenicio a Mozia: nuovi dati dall'Area sacra del Kothon: A. Lemaire (ed.), Phéniciens d'Orient et d'Occident. Mélanges Josette Elayi (Cahiers de l'Institut du Proche-Orient ancien du Collège de France II), Paris 2014, pp. 491-504.

2014b The so-called "Kothon" at Motya. The sacred pool of Baal 'Addir/Poseidon in the light of recent archaeological investigations by Rome La Sapienza University - 2005-2013. Stratigraphy, architecture, and finds (Quaderni di Archeologia Fenicio-Punica/Colour Monograph 03), Roma 2014.

2015a Temples in Motya and their Levantine prototypes: Phoenician religious architectural tradition: C. DOUMET-SERHAL (ed.), Cult and ritual on the Levantine Coast and its impact on the Eastern Mediterranean Realm. Proceedings of the International Symposium, Beirut 2012 (Bulletin d'Archéologie et d'Architecture Libanaises, Hors-Série, X), Beirut 2015, pp. 83-108.

2015b Mozia tra VI e V secolo a.C. Monumentalizzazione e organizzazione socio-politica: un nuovo modello: M.P. BAGLIONE - L.M. MicheTti (a cura di), Le lamine d'oro a cinquant'anni dalla scoperta. Dati archeologici su Pyrgi e rapporti con altre realtà del Mediterraneo (Scienze dell’Antichità 21.2), Roma 2015, pp. 225-245.

NigRo, L. (ed.)

2004 Mozia - X. Rapporto preliminare della XXII campagna di scavi - 2002 condotta congiuntamente con il Servizio Beni Archeologici della Soprintendenza Regionale per i Beni Culturali e Ambientali di Trapani (Quaderni di Archeologia Fenicio-Punica I), Roma 2004.

2005 Mozia - XI. Il Tempio del Kothon. Rapporto preliminare delle campagne di scavi XXIII e XXIV (2003-2004) condotte congiuntamente con il Servizio Beni Archeologici della Soprintendenza Regionale per i Beni Culturali e Ambientali di Trapani (Quaderni di Archeologia Fenicio-Punica II), Roma 2005.

2007 Mozia - XII. Zona D. La "Casa del sacello domestico", il "Basamento meridionale e il Sondaggio stratigrafico I. Rapporto preliminare delle campagne di scavi XXIII e XXIV (2003-2004) condotte congiuntamente con il Servizio Beni Archeologici della Soprintendenza Regionale per i Beni Culturali e Ambientali di Trapani (Quaderni di Archeologia Fenicio-Punica, III), Roma 2007.

2011 Mozia - XIII. Zona F. La Porta Ovest e la Fortezza Occidentale. Rapporto preliminare delle campagne di scavi XXIII-XXVII (2003-2007) condotte congiuntamente con il 
Servizio Beni Archeologici della Soprintendenza Regionale per i Beni Culturali e Ambientali di Trapani (Quaderni di Archeologia Fenicio-Punica VI), Roma 2011.

NigRo, L. - SPAGNOLI, F.

2012 Alle sorgenti del Kothon. Il rito a Mozia nell'Area sacra di Baal 'Addir - Poseidon. Lo scavo dei pozzi sacri nel Settore C Sud-Ovest (2006-2011) (Quaderni di Archeologia Fenicio-Punica/Colour Monograph 02), Roma 2012.

ORSINGHER, A

2011 La ceramica punica del IV secolo a.C. dalla Fortezza Occidentale: L. NigRo (a cura di), Mozia - XIII. Zona F. La Porta Ovest e la Fortezza Occidentale. Rapporto preliminare delle campagne di scavi XXIII-XXVII (2003-2007) condotte congiuntamente con il Servizio Beni Archeologici della Soprintendenza Regionale per i Beni Culturali e Ambientali di Trapani (Quaderni di Archeologia Fenicio-Punica VI), Roma 2011, pp. 112-131.

Pelagatti, P.

1965 Antefisse sileniche siceliote: Cronache di archeologia 4 (1965), pp. 79-98.

SCHIAVO, D.

$1756 \quad$ Memorie per servire alla storia letteraria di Sicilia. Tomo secondo, Palermo 1756.

SPAGNOLI, F.

2013 Demetra a Mozia: evidenze dall’Area sacra del Kothon nel V secolo a.C.: Vicino Oriente XVII (2013), pp. 153-164.

SPATAFORA, F. - GANDOLFO, L. (a cura di)

2014 "Del Museo di Palermo e del suo avvenire”: il Salinas ricorda Salinas, 1914-2014, Palermo 8 luglio - 4 novembre 2014, Palermo 2014.

TотI, M.P.

2008 Le «collezioni» del Museo G. Whitaker di Mozia: E. ACQUARO - D. FERRARI (a cura di), Le antichità fenicie rivisitate. Miti e culture (Biblioteca di Byrsa 5), Lugano 2008, pp. 6982.

UBERTI, M.L.

1997 L’artigianato: Z. CHERIF, Terres cuites puniques de Tunisie (Corpus delle antichità fenicie e puniche), Roma 1997, pp. 163-217.

UHLENBROCK, J.P.

2002 La coroplastica nella Sicilia orientale e meridionale nell'età dei due Dionisi: problemi di stile e cronologia archeologica: N. BonACASA - L. BRACCESI - E. DE Miro (a cura di), La Sicilia dei due Dionisi. Atti della settimana di studio, Agrigento 24-28 febbraio 1999 VASSALLO, S. (Progetto Akragas 2), Roma 2002, pp. 321-337.

2005 Himera. Città greca. Guida alla storia e ai monumenti, Palermo 2005.

WIEDERKEHR SCHULER, E.

2004 Les protomés féminines du sanctuaire de la Malophoros à Sélinonte (Cahiers du Centre Jean Bérard XXII), Naples 2004.

WINTER, F.

1903a Die antiken Terrakotten. Die Typen der figürlichen Terrakotten, III, 1, Berlin 1903.

1903b Die antiken Terrakotten. Die Typen der figürlichen Terrakotten, III, 2, Berlin 1903. 


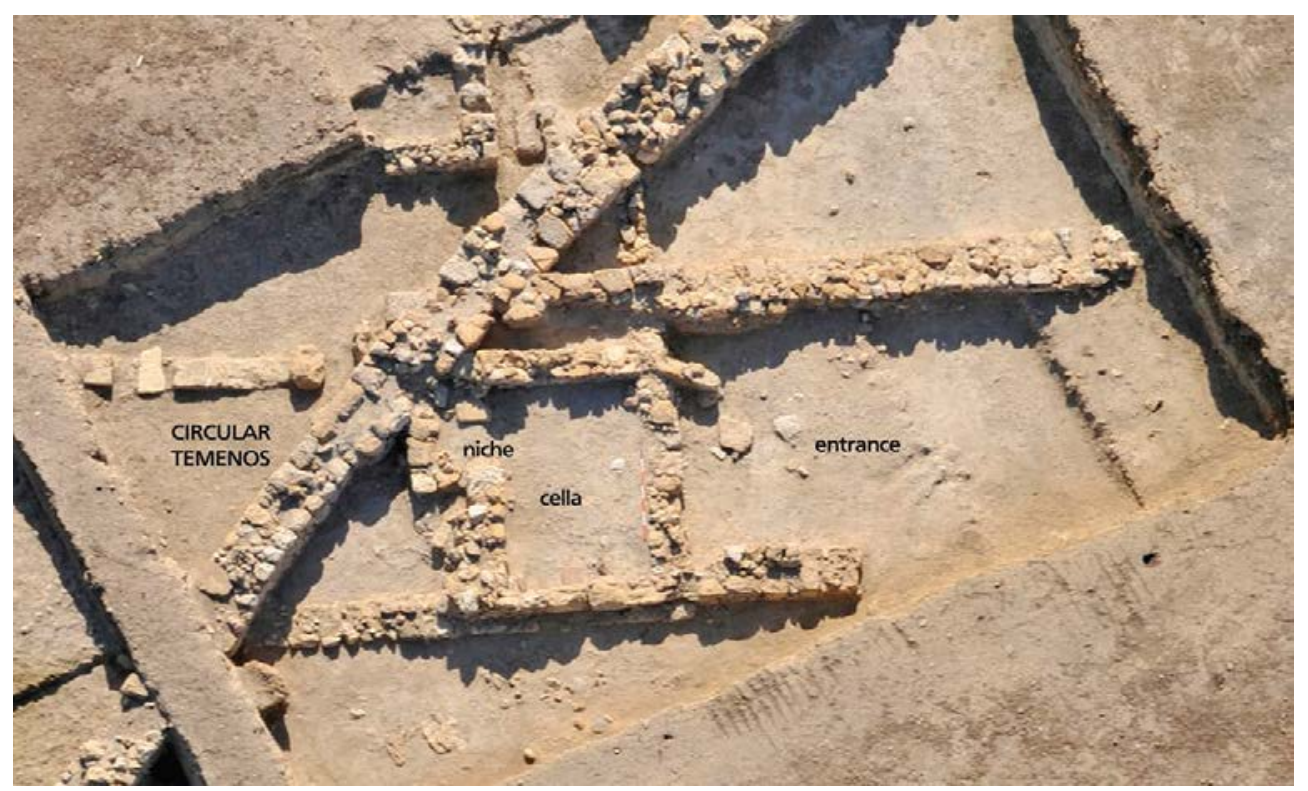

Fig. 1 - Mozia, Zona C: Veduta aerea del Sacello C6-C4 nell’Area sacra del Kothon (da Nigro 2015, fig. 14).
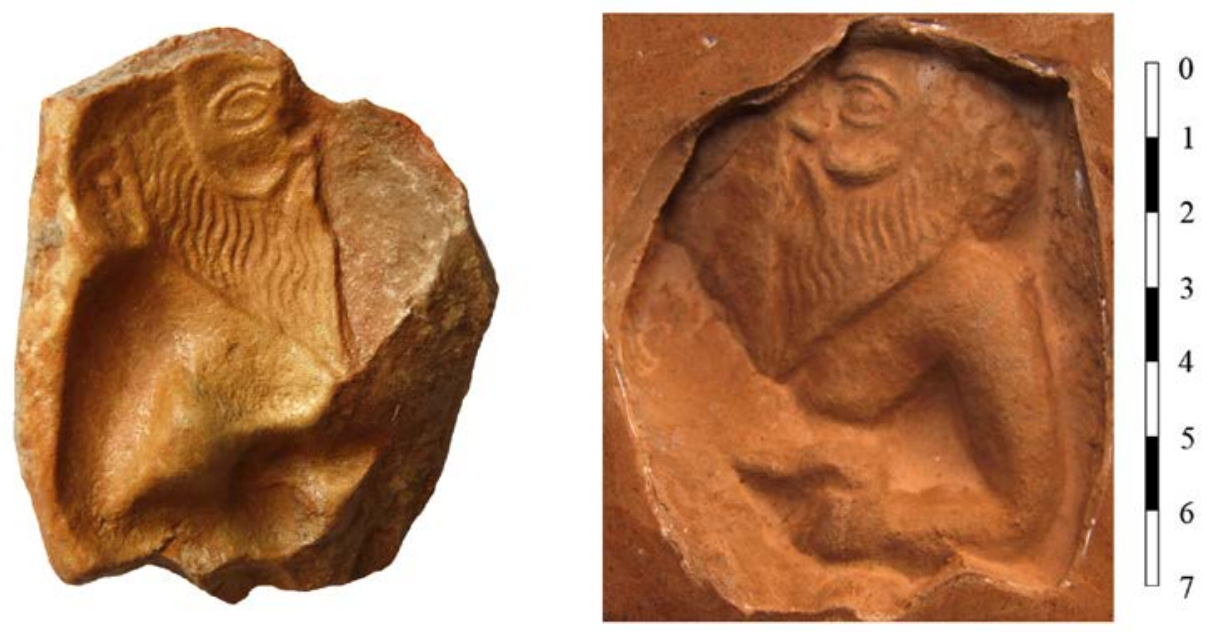

Fig. 2 - Mozia, Zona C: la matrice MC.05.396 e il relativo calco in gesso (ㄷ Missione Archeologica a Mozia, Università “la Sapienza” di Roma, foto dell’autore). 


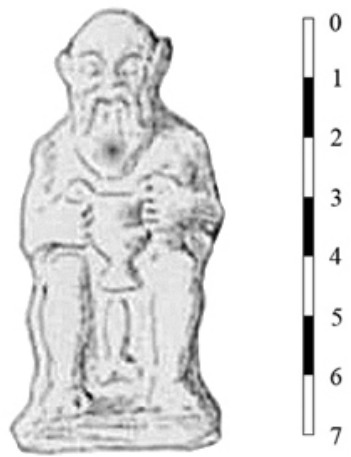

Fig. 3 - Sileno con kantharos (da Winter 1903a, 218, n. 2).

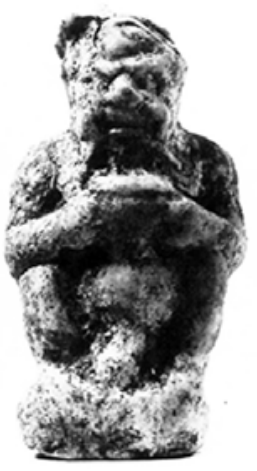

Fig. 4 - Roma: sileno con coppa (da Besques 1982, fig. 10).

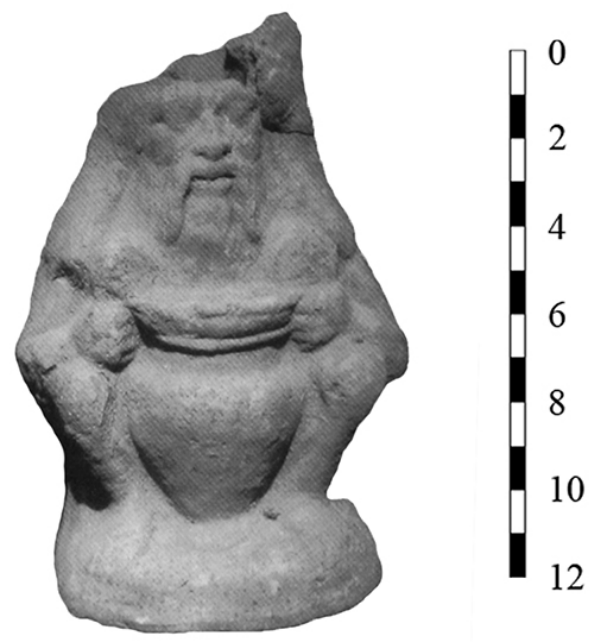

Fig. 5 - Cartagine: sileno con cratere (da Cherif 1997, tav. XXVII). 


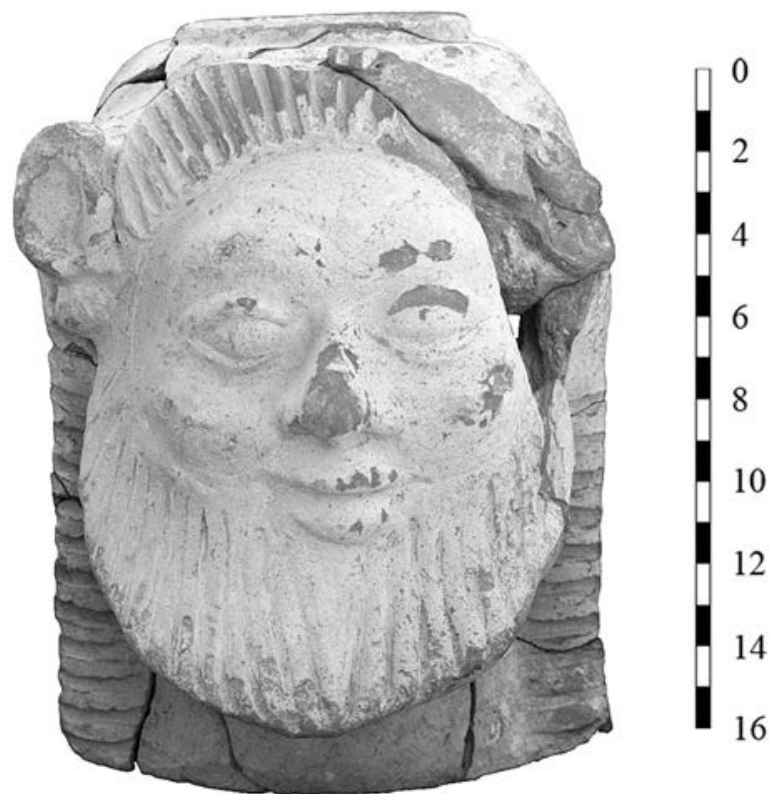

Fig. 6 - Mozia, Zona E: testa di sileno in terracotta (da Famà - Toti 2000, tav. XC:1a).

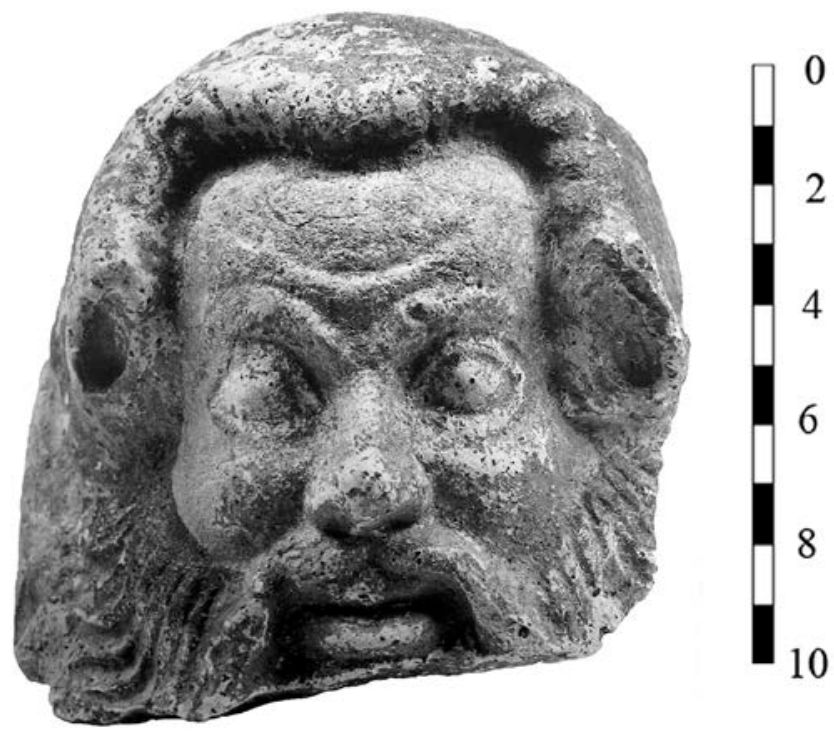

Fig. 7 - Naxos: antefissa silenica (da Epifanio 1990, 255). 

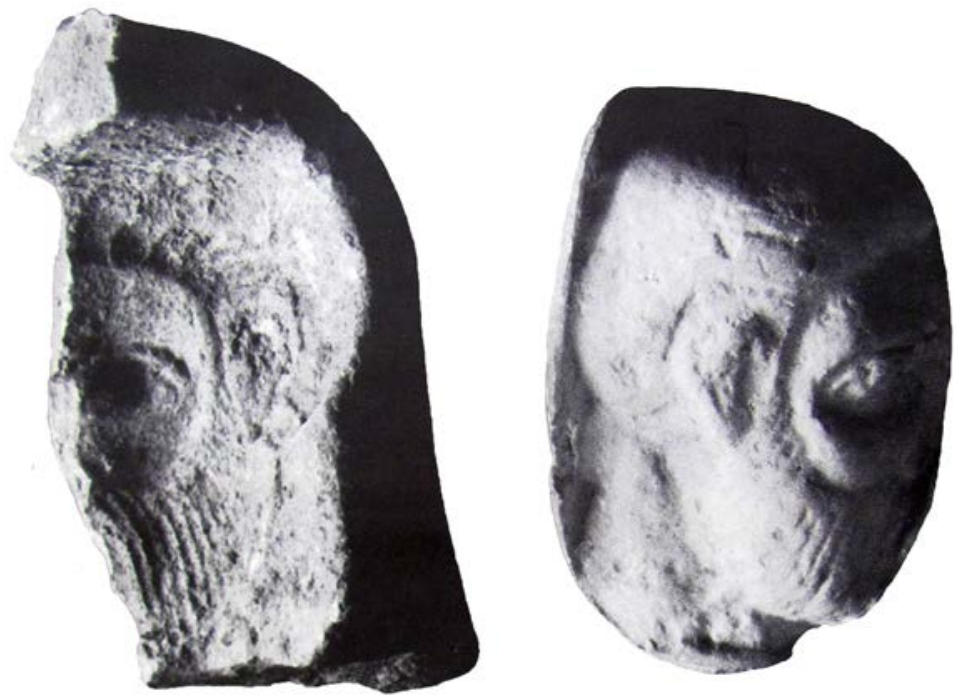

Fig. 8 - Ibiza: frammento di matrice e calco (da Almagro Gorbea 1980, tav. CCII:1-2). 


\section{SOMMARIO}

DipartimENTo SCIENZE DELl'ANTICHITÀ

SEZIONE di ORIENTALISTICA

\section{VICINO ORIENTE \\ XIX - 2015}

L. Nigro - Bethlehem in the Bronze and lron Ages
in the light of recent discoveries by the Palestinian MOTA-DACH

$\checkmark$. Pisaniello - Parallel passages among Hittite-Luwian rituals:

for the restoration of $K U B 35.146$

F. Spagnoli - Una testa di sileno in bronzo da Mozia

N. Chiaren

dall Area sacra del Kothon a Mozia

G. Labisi - al-Fudayn: an Umayyad residence in Northern Jordan

P. Buzi - Early Christianity in the Fayyūm: the new contribution of archaeology

I. Materia - Preliminary notes on the ware depicted on the ceiling

of the Cappella Palatina in Palermo

S. Autiero - Indian Ocean trade:

a reassessment of the pottery find
$\left(3^{\text {rd }}\right.$ century $B C-S^{S^{t h}}$ century $\left.A D\right)$

M.M. Jamhawi - N. Al-Shakarchi - I. Al-Hashimi

Assessment of tourists' satisfaction in the downtown of Amman

SCAVI E RICERCHE

L. Nigro - C. Fiaccavento - M. Jaradat - J. Yasine

A

L. Nigro - D. Montanari - M. Ghayyada - J. Yasine

A Middle Bronze and Iron Age necropolis near Bethlehem (Palestine)

L. Nigro - G. Ripepi - I. Hamdan - J. Yasine

15 Interim Report

and valorization of archaeological heritage

R. Francia - L'archivio di tavolette del complesso B-C-H di Büyükkale

organizazione degli archivi reali ittiti. Considerazioni preliminari

V. Pisaniello - La collezione di tavolette del complesso B-C-H di Büyükkale

T. De Vincenzi - L'archivio di tavolette del complesso B-C-H
sull'acropoli di Büyükkale

Museo del Vicino Oriente, Egitto e Mediterraneo

L. Nigro - Il nuovo allestimento del Museo del Vicino Oriente,

Egitto e Mediterraneo della Sapienza

D. Montanari - Bollettino delle attività del Museo del Vicino Oriente,

Egito e Meditraneo della Sapienza, anno 2015

RECENSIONI

A. Orsingher - E. PAPPA (2013), Early Iron Age Exchange in the West:

(Ancient Near Eastern Studies Supplement Series 43)

Leuven - Paris - Walpole 2013, MA.: Peeters $\frac{1}{2}$

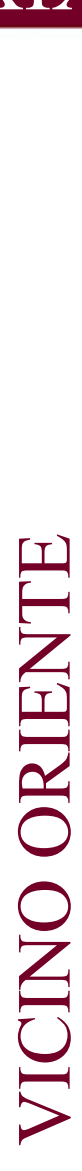

345

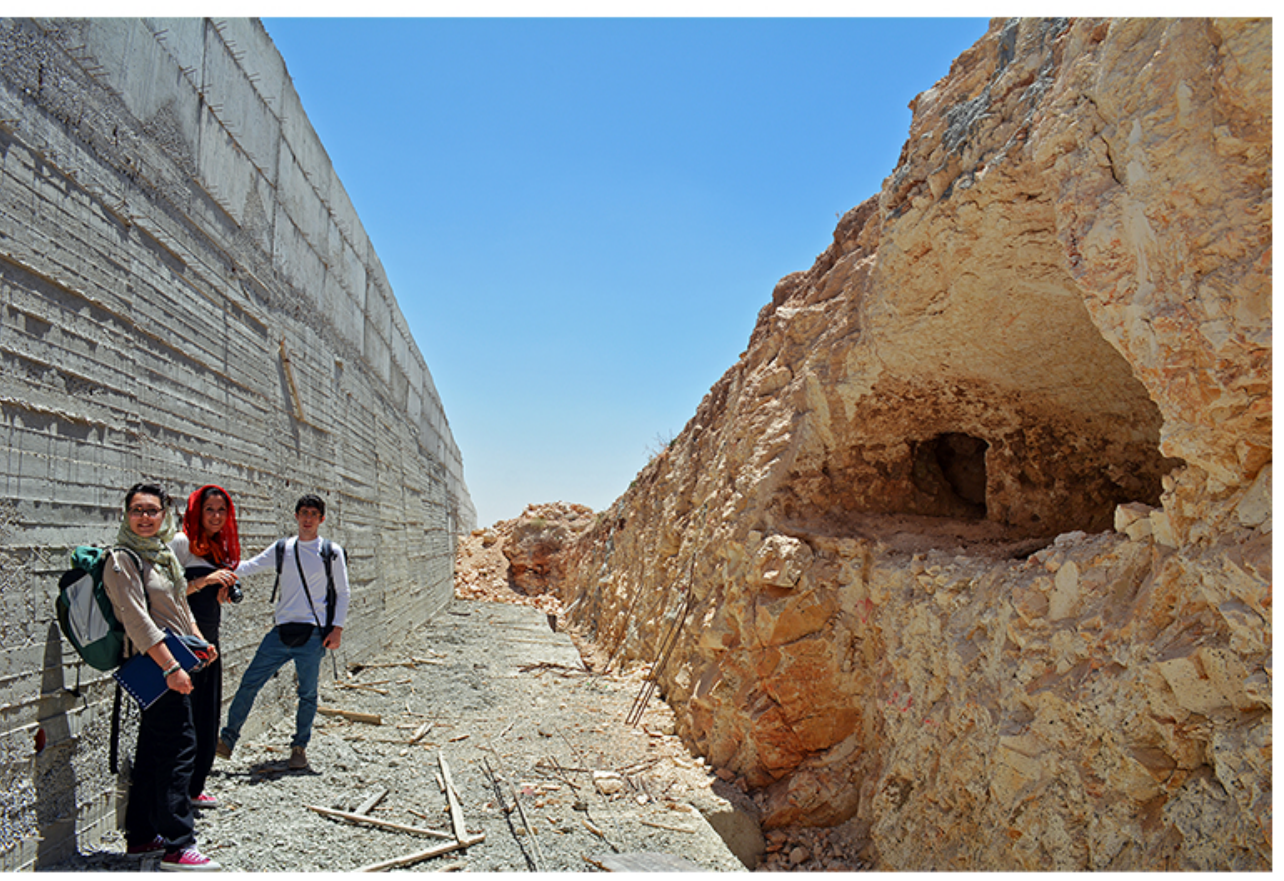

ROMA 2015 


\section{VICINO ORIENTE XIX - 2015}


VICINO ORIENTE

SAPIENZA UNIVERSITÀ DI ROMA

DIPARTIMENTO SCIENZE DELL'ANTICHITÀ

SEZIONE DI ORIENTALISTICA

Scientific Editor: Lorenzo Nigro

International Scientific Committee: Brian Rose, Frank Braemer, Mounir Fantar, Piero Bartoloni, Thomas Schaefer, Zeidan Kafafi

National Scientific Committee: Carlo Giovanni Cereti, Maria Vittoria Fontana, Sebastiano Tusa, Massimiliano Marazzi

Editorial Board: Daria Montanari, Chiara Fiaccavento

Tipografia: SK7 - Roma

ISSN 0393-0300

Rivista con comitato di referee

Journal with international referee system

www.lasapienzatojericho.it/SitoRivista/Journal/Rivista.php

In copertina: Tomba B9, necropoli di Khalet al-Jam’a (Betlemme). 
VICINO ORIENTE

SAPIENZA UNIVERSITÀ DI ROMA

DIPARTIMENTO SCIENZE DELL'ANTICHITÀ

SEZIONE DI ORIENTALISTICA

\section{SOMMARIO}

ARTICOLI

L. Nigro - Bethlehem in the Bronze and Iron Ages

in the light of recent discoveries by the Palestinian MOTA-DACH

V. Pisaniello - Parallel passages among Hittite-Luwian rituals:

for the restoration of KUB 35.146

F. Spagnoli - Una testa di sileno in bronzo da Mozia

N. Chiarenza - Una matrice per terrecotte con sileno dall'Area sacra del Kothon a Mozia

G. Labisi - al-Fudayn: an Umayyad residence in Northern Jordan

P. Buzi - Early Christianity in the Fayyūm: the new contribution of archaeology

I. Materia - Preliminary notes on the ware depicted on the ceiling

of the Cappella Palatina in Palermo

S. Autiero - Indian Ocean trade:

a reassessment of the pottery finds from a multidisciplinary point of view ( $3^{\text {rd }}$ century $B C-5^{\text {th }}$ century $\left.A D\right)$

M.M. Jamhawi - N. Al-Shakarchi - I. Al-Hashimi

Assessment of tourists' satisfaction in the downtown of Amman

SCAVI E RICERCHE

L. Nigro - C. Fiaccavento - M. Jaradat - J. Yasine Archaeology from A to Z: Abu Zarad, an ancient town in the heartland of Palestine

L. Nigro - D. Montanari - M. Ghayyada - J. Yasine

Khalet al-Jam'a. A Middle Bronze and Iron Age necropolis near Bethlehem (Palestine) 185 
VICINO ORIENTE

SAPIENZA UNIVERSITÀ DI ROMA

DIPARTIMENTO SCIENZE DELL'ANTICHITÀ

SEZIONE DI ORIENTALISTICA

L. Nigro - G. Ripepi - I. Hamdan - J. Yasine

The Jericho Oasis Archaeological Park - 2015 Interim Report.

Italian-Palestinian Cooperation for protection

and valorization of archaeological heritage

R. Francia - L'archivio di tavolette del complesso B-C-H di Büyükkale

e l'organizzazione degli archivi reali ittiti. Considerazioni preliminari

V. Pisaniello - La collezione di tavolette del complesso B-C-H di Büyükkale

T. De Vincenzi - L'archivio di tavolette del complesso B-C-H sull'acropoli di Büyükkale

Museo del Vicino ORIente, Egitto e MediterRaneo

L. Nigro - Il nuovo allestimento del Museo del Vicino Oriente,

Egitto e Mediterraneo della Sapienza

D. Montanari - Bollettino delle attività del Museo del Vicino Oriente,

Egitto e Mediterraneo della Sapienza, anno 2015

\section{RECENSIONI}

A. Orsingher - E. PAPPA (2013), Early Iron Age Exchange in the West:

Phoenicians in the Mediterranean and the Atlantic

(Ancient Near Eastern Studies Supplement Series 43),

Leuven - Paris - Walpole 2013, MA.: Peeters 